\title{
A Method for Investigation of Size-Dependent Protein Binding to Nanoholes Using Intrinsic Fluorescence of Proteins
}

\author{
Bita Malekian, ${ }^{\dagger, \|, \perp}$ Ivan Maximov, ${ }^{\dagger, \|}$ Rainer Timm, ${ }^{\S, \| \odot ~}$ Tommy Cedervall, ${ }^{\ddagger}, \|$ and Dan Hessman ${ }^{*},+, \|_{\odot}$ \\ ${ }^{\dagger}$ Solid State Physics, ${ }^{\ddagger}$ Biochemistry and Structural Biology, ${ }^{\S}$ Synchrotron Radiation Research, and ${ }^{\|}$NanoLund, Lund University, P.O. \\ Box 118, 22100 Lund, Sweden
}

\section{Supporting Information}

ABSTRACT: We have developed a novel method to study the influence of surface nanotopography on human fibrinogen adsorption at a given surface chemistry. Well-ordered arrays of nanoholes with different diameters down to 45 $\mathrm{nm}$ and a depth of $50 \mathrm{~nm}$ were fabricated in silicon by electron beam lithography and reactive ion etching. The nanostructured chip was used as a model system to understand the effect of size of the nanoholes on fibrinogen adsorption. Fluorescence imaging, using the intrinsic fluorescence of proteins, was used to characterize the effect of the nanoholes on fibrinogen adsorption. Atomic force microscopy was used as a complementary technique for further characterization of the interaction. The results demonstrate that as the size of the nanoholes is reduced to $45 \mathrm{~nm}$, fibrinogen adsorption is significantly increased.
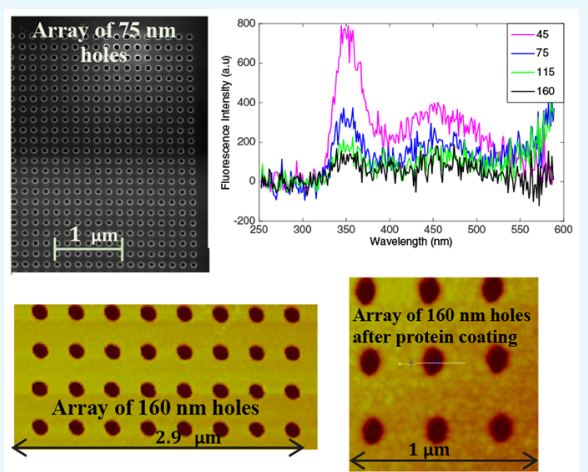

\section{INTRODUCTION}

The interaction of proteins with artificial materials is of great importance in different areas, such as medical applications and nanosafety. The biocompatibility of implanted materials largely depends on the first interactions occurring at the interface between the material's surface and a biological system. ${ }^{1}$ Protein adsorption, which takes place spontaneously, is the first event in a series of biological responses that occur when an artificial material comes into contact with biological environments. ${ }^{1,2}$ The adsorbed protein layer on the surface of the material is often important in determining subsequent biological responses, such as cell adhesion and immunological response., ${ }^{2,3}$ It is well known that the surface nanotopography of artificial materials influences protein adsorption when the size of the nanostructures is comparable to protein dimensions. ${ }^{1,4}$ Therefore, more knowledge of the effect of surface nanotopography on protein binding is needed to better understand the interaction between surface nanotopography and protein binding in situations when the human body is, unintentionally, exposed to nanomaterials in the lab, or from the use of commercial products, or when nanomaterials and medical implants are used intentionally as a tool for diagnosis or treatment.

Different nanoscale features can be intentionally present on the surface of artificial materials, or be present due to materials defects, or because the biological environment with time changes the surface. These nanoscale features can have both positive and negative curvatures. The effect of positive curvature on protein response has been extensively studied using spherical nanoparticles, demonstrating that protein binding increases with the diameter of nanoparticles. ${ }^{5-7}$ However, much less attention has been paid in the literature to the effect of negative curvature or nanoholes on protein binding. Therefore, more systematic studies are required to better understand the influence of negative curvature on protein adsorption.

Nanofabrication technology, developed originally for the electronics industry, enables the creation of well-defined and highly reproducible nanostructured surfaces and opens up the possibility of studying the influence of such surfaces on protein behavior. Specifically, introducing nanoscale features onto the materials surfaces provides the opportunity to create new types of nanoscale features, not readily available when using spherical nanoparticles. In particular, this approach allows for the creation of negative curvatures, such as nanoholes, nanogrooves, and nanopyramids. ${ }^{8,9}$ Moreover, constructing nanostructured surfaces on macroscopic substrates provides a more convenient platform to employ and combine surface sensitive characterization techniques, such as X-ray photoelectron spectroscopy (XPS), atomic force microscopy (AFM), and fluorescence spectroscopy in a new manner.

This article describes a novel method to investigate the effect of surface nanotopography on protein binding. Our approach includes the fabrication of highly controllable nanostructured surfaces, deposition of a monolayer of proteins on the nanostructured area and characterization using fluorescence and AFM techniques. We have used state-of-the-art nanofabrication techniques to produce well-ordered arrays of nanoholes in silicon $(\mathrm{Si})$ substrates with high reproducibility to study the effect of size/diameter of the nanoholes on protein

Received: March 1, 2017

Accepted: May 3, 2017

Published: August 22, 2017 

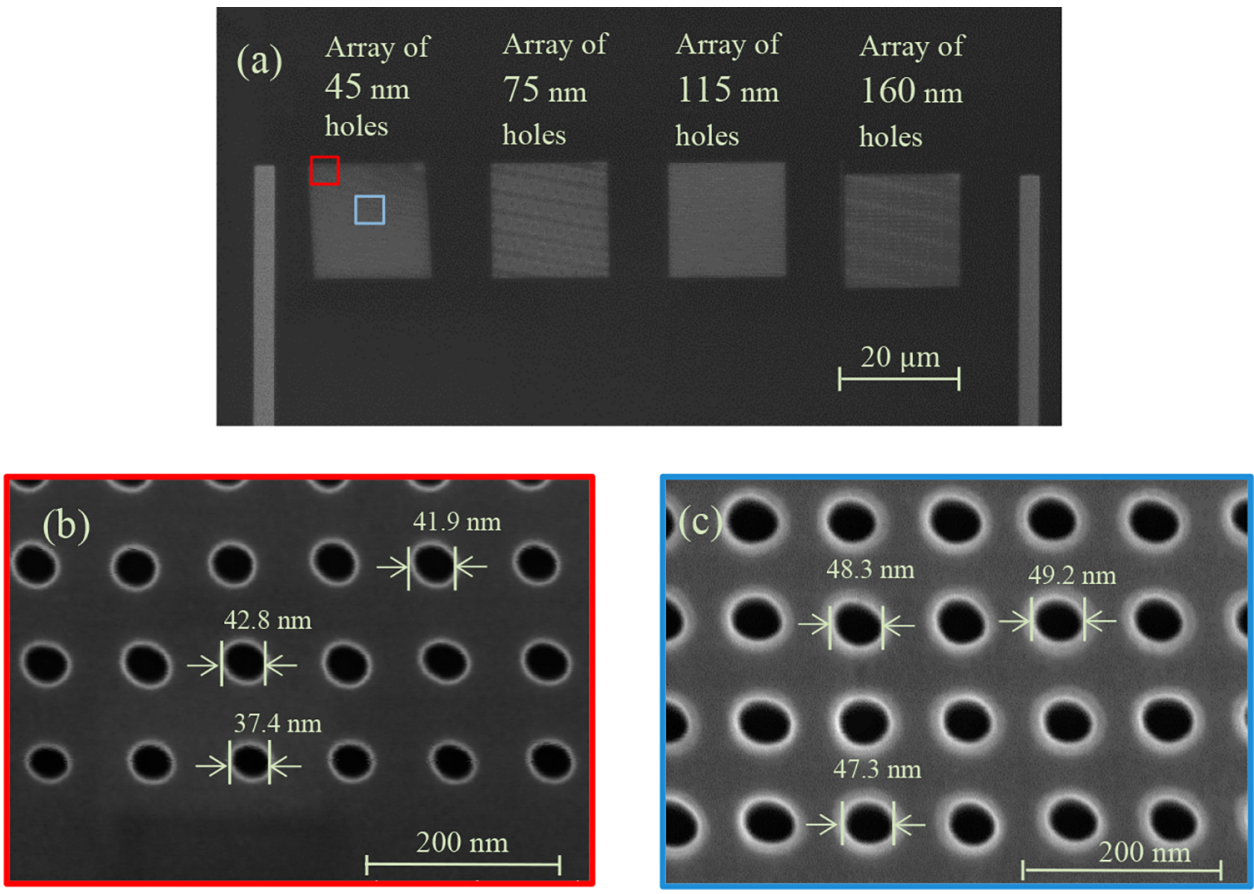

Figure 1. SEM images of (a) the arrays of nanoholes fabricated in four distinct regions in the Si substrate before etching, each region containing an array of nanoholes with a specific diameter. (b, c) The magnified views of the array of $45 \mathrm{~nm}$ holes after etching in the edge and in the center of the array, respectively.

binding. Arrays of nanoholes with different diameters down to $45 \mathrm{~nm}$ and a depth of $50 \mathrm{~nm}$ were created in Si substrates by electron beam lithography (EBL) and reactive ion etching (RIE). In this study, we have used human fibrinogen and, in particular, used the intrinsic fluorescence of the proteins to obtain information about the influence of size of the nanoholes on protein adsorption. Fluorescence imaging using the intrinsic fluorescence of proteins, instead of labeling with fluorophores, provides us the opportunity to observe how protein behavior is influenced by surface nanotopography without being affected by the presence of fluorophores. In addition, the same label-free proteins can be used in different characterization techniques by avoiding extrinsic fluorophores. The distribution of the adsorbed fibrinogen molecules onto the nanostructured area was also probed by AFM. The result of this initial study reveals a stronger binding tendency of human fibrinogen to the smallest nanoholes compared with the flat surface around and also compared to other sizes of nanoholes. Our results suggest that using intrinsic fluorescence of proteins combined with AFM is a promising approach to study the influence of surface nanotopography on protein binding.

\section{RESULTS AND DISCUSSION}

We have fabricated Si model substrates consisting of arrays of nanoholes, deposited a monolayer of protein molecules on the model substrates, and used fluorescence and AFM techniques to characterize the influence of nanoholes with different diameters on protein adsorption. EBL and RIE were used to produce the nanostructured $\mathrm{Si}$ substrates. The diameter and depth of the arrays of nanoholes were characterized by scanning electron microscopy (SEM) and AFM, respectively.

Figure 1a shows the SEM image of the four arrays of nanoholes, which were patterned on the Si substrate by EBL. The four distinct nanostructured regions were fabricated on the Si substrate by EBL and RIE, each region containing an array of nanoholes with a specific diameter. The four arrays of nanoholes were created with diameters of typically 45,75 , 115 , and $160 \mathrm{~nm}$ and pitches of 60, 75, 85, and $140 \mathrm{~nm}$, respectively. The array of nanoholes with a certain diameter, for example, $45 \mathrm{~nm}$, was fabricated in an area of $20 \times 20 \mu \mathrm{m}^{2}$ (Figure 1). The space between two adjacent nanostructured regions was $10 \mu \mathrm{m}$. The sizes of the nanoholes are small enough to expect an influence on the protein used in this study because the sizes are comparable to the hydrodynamic diameter of the proteins. In this work, we have used human fibrinogen with a hydrodynamic diameter of $22 \mathrm{~nm} .{ }^{10}$

Figure 1b,c displays SEM images of the nanoholes close to the edge and in the center of the array of $45 \mathrm{~nm}$ holes after etching and removing the e-beam resist. There is a variation of $12 \mathrm{~nm}$ in diameter in the arrays of the $45 \mathrm{~nm}$ holes from the edges of the region toward the center, which might be due to the proximity effect in the EBL process. ${ }^{11}$ The minimum diameter (at the top and bottom of the array of $45 \mathrm{~nm}$ holes) and the maximum diameter (at the center of the array) were 36 and $49 \mathrm{~nm}$, respectively. There was no difference in the diameter obtained by SEM imaging before and after the etching (and removing the e-beam resist). Therefore, to prevent the possible contamination of the substrate surface with carbon deposition during the SEM imaging, the nanostructured substrates, which were used for protein coating were characterized by SEM only before the etching.

Fluorescence imaging, using intrinsic fluorescence of proteins, is the main characterization technique in our work, revealing size-dependent fibrinogen adsorption to the nanoholes. In this work, it is essential to form only a monolayer of proteins on the $\mathrm{Si}$ surface because only the interaction of protein molecules that are in direct contact with the surface is of interest. Furthermore, to compare protein adsorption between the arrays of nanoholes with different diameters, the 
(a)

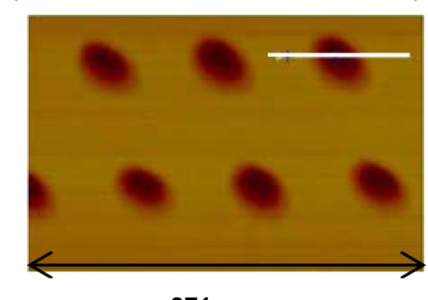

(c)

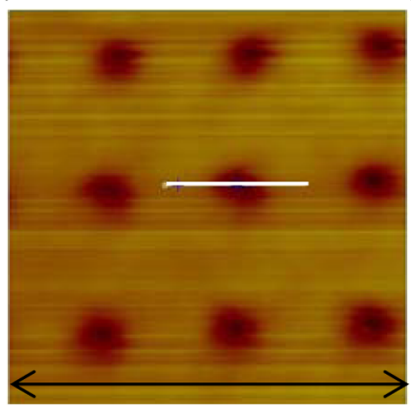

$337 \mathrm{~nm}$ (b)

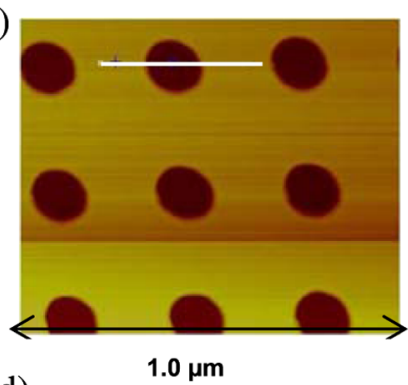

(d)

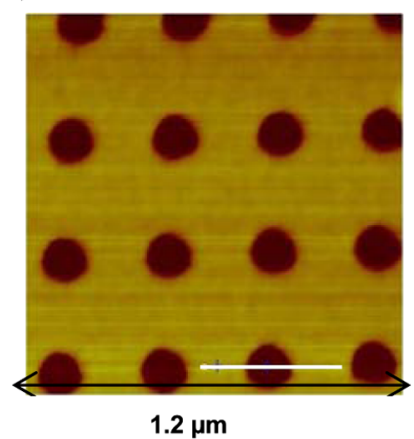

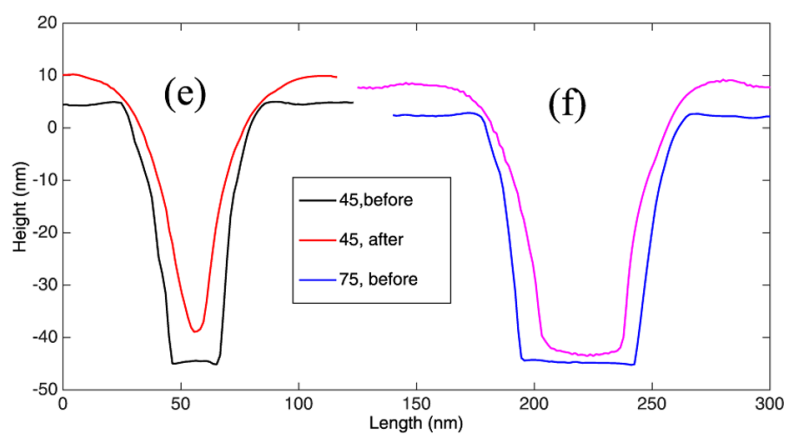

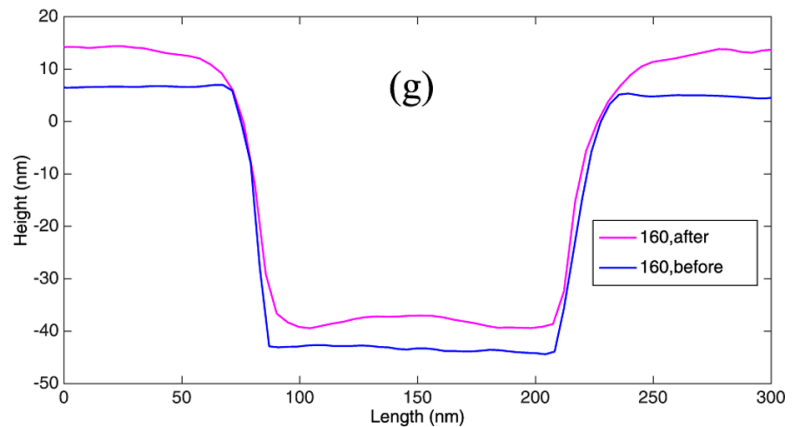

Figure 2. AFM images and cross-sectional cuts through random nanoholes. (a-d) AFM images of the regions containing the arrays of $45 \mathrm{~nm}$ (a, c) and $160 \mathrm{~nm}(\mathrm{~b}, \mathrm{~d})$ holes before $(\mathrm{a}, \mathrm{b})$ and after (c, d) firbrinogen deposition. The white line passing through the nanoholes corresponds to the crosssectional cuts shown in (e) and (g). (e, f, g) AFM cross-sectional cuts through random nanoholes (the corresponding nanoholes shown in (a)-(d)) before and after protein deposition with arbitrary shift (e) $45 \mathrm{~nm}$ holes, (f) $75 \mathrm{~nm}$ holes, and (g) $160 \mathrm{~nm}$ holes. Note that different nanoholes were scanned before and after protein coating.

protein coverage should be homogeneous, at least in the nanostructured area.

We could deposit a uniform monolayer of fibrinogen molecules on the flat/unprocessed Si surface by immersing the $\mathrm{Si}$ substrates in a protein solution with suitable incubation and rinsing times. The 5 min' incubation of the Si substrate in the fibrinogen solution, followed by $1 \mathrm{~min}$ rinsing in water, and drying with nitrogen gas, resulted in deposition of a homogeneous monolayer of human fibrinogen on the flat $\mathrm{Si}$ surface. XPS data analysis of the unprocessed Si substrate with deposited fibrinogen molecules indicated a thickness of 4-5 $\mathrm{nm}$, which corresponds to monolayer coverage by the fibrinogen molecules (see Supporting Information 1). The typical dimensions of the rodlike shaped fibrinogen molecules, when in solution, are $45 \mathrm{~nm} \times 9 \mathrm{~nm} \times 6 \mathrm{~nm}$. ${ }^{12}$ Consequently, the thickness obtained by XPS indicates that fibrinogen molecules are lying down on the unprocessed Si surface.

The distribution of the adsorbed fibrinogen molecules in the nanostructured regions was investigated by AFM. The AFM measurements were conducted in tapping mode using super sharp tips. Using tips with high aspect ratio reduces the tip convolution artefacts and was critical for this work. ${ }^{13}$ Because the smallest diameter of the nanoholes is $45 \mathrm{~nm}$, the tip should have a high aspect ratio to reach the bottom of the nanoholes and provide a reliable profile. The depth measured by the AFM was about $50 \mathrm{~nm}$ for all different sizes of the nanoholes with a variation of $5 \mathrm{~nm}$ between different positions in the nanostructured area. Probing nanoholes by super sharp tips allowed us to compare the sidewall profile of the nanoholes before and after protein deposition. Because the measured nanohole profile is influenced by the shape of the AFM tip, the nanoholes were probed before and after fibrinogen deposition by the same tip to ensure that any differences before and after protein coating are due to the presence of the protein molecules and not due to the difference between different AFM tips. It should be mentioned, that based on the half-cone angle of the super sharp AFM tip, the tip is sharper than the measured slope of the nanohole sidewalls. Therefore, it can be expected that the slope of the nanohole sidewalls in AFM crosssectional cuts is close to the actual shape of the nanoholes.

Figure $2 \mathrm{a}-\mathrm{d}$ displays AFM images of the regions containing $45 \mathrm{~nm}$ (Figure 2a,c) and $160 \mathrm{~nm}$ (Figure 2b,d) nanoholes before (Figure 2a,b) and after (Figure 2c,d) protein deposition. The corresponding cross-sectional measurements are shown in Figure $2 \mathrm{e}, \mathrm{g}$. It was not possible to find the same nanohole with the AFM before and after protein coating. Therefore, the crosssectional cuts in, for instance, Figure $2 \mathrm{f}$ were obtained from two different nanoholes but with the same nominal size. Note that there is no absolute height scale in the cross-sectional measurements shown in Figure $2 \mathrm{e}-\mathrm{g}$.

The cross-sectional measurements in Figure $2 \mathrm{e}-\mathrm{g}$ clearly display rounded corners at the top of the nanoholes after protein coating, which is due to the effect of bound fibrinogen molecules. In other words, there is a strong interaction at the top corner of the nanoholes with proteins, which seems to be independent of the nanoholes' size. Comparing AFM crosssectional measurements before and after protein deposition also indicates that fibrinogen molecules are present inside the nanoholes. The cross-sectional measurements in Figure 2 show that the width of the nanoholes is reduced after protein deposition indicating that fibrinogen molecules were adsorbed to the sidewalls, particularly to the sidewalls of the arrays of the 45 and $75 \mathrm{~nm}$ holes (Figure 2e,f) (see Supporting Information 2). On average, the cross-sectional measurements show a similar depth before and after protein deposition, demonstrating the same amount of protein binding to the bottom of the nanoholes as to the flat area in between the nanoholes. The difference in depth between the cross sections in Figure 2 is 
attributed to the $5 \mathrm{~nm}$ variation in the etched depth discussed before.

Control measurements were performed with a standard AFM tip, where the same tip was used to first image nanoholes after protein coating, showing a reduced nanohole width with rounded corners, and then image a reference sample without proteins, showing a larger nanohole width with steeper corners. Thus, we can be sure that the change in AFM cross section observed after protein deposition is due to the protein molecules attached to the surface and not due to contamination of the tip. It should be noted that the interaction of proteins with the AFM tip is very complex, which should be considered in all AFM data interpretation.

It seems in Figure 2e that the AFM tip could not reach the bottom of the $45 \mathrm{~nm}$ holes after protein coating, apparently due to the interaction with the fibrinogen molecules adsorbed to the sidewalls. However, cross-sectional measurements in other nanoholes with the same nominal size show that the tip could scan the bottom of the $45 \mathrm{~nm}$ holes (see Supporting Information 2). In conclusion, AFM cross-sectional measurements demonstrate that the width of the nanoholes is significantly reduced after protein coating in the array of 45 and $75 \mathrm{~nm}$ holes, indicating that fibrinogen molecules were adsorbed to the sidewalls of the nanoholes. In addition, no significant difference in the height of the nanoholes is observed before and after protein deposition. The same height difference before and after protein coating also indicates that the same amount of fibrinogen molecules is adsorbed at the bottom of the nanoholes as to the flat area in between the nanoholes.

We have used fluorescence imaging using the intrinsic fluorescence of proteins to compare the amount of fibrinogen molecules adsorbed in the nanoholes with different diameters. The intrinsic fluorescence is the fluorescence emitted by many proteins when excited with UV light. ${ }^{14}$ Among the 20 amino acids in proteins, only 3 of them, tryptophan (Trp), tyrosine (Tyr), and phenylalanine (Phe), provide fluorescence emission upon exposure to UV light. ${ }^{15}$ The emission spectrum of Trp is often changed in response to changes in its local environment, such as conformational transitions and substrate binding. ${ }^{15}$ Therefore, variation in the intensity of $\operatorname{Trp}$ emission or observation of any shift in the Trp peak provides information about the influence of nanoholes on protein binding. We used the emission intensity of $\operatorname{Trp}$ as an indication of the proteins' presence on the surface. The emission maximum of Trp occurs at $350 \mathrm{~nm}$ when it is excited at $280 \mathrm{~nm} .{ }^{16}$ We have designed a fluorescence setup sensitive enough to detect the Trp emission of a monolayer of fibrinogen molecules adsorbed on the $\mathrm{Si}$ surface under dry conditions. Figure 3 shows the fluorescence emission of the fibrinogen layer adsorbed to the Si substrate recorded by our setup. It should be noticed that the Trp emission typically dominates the intrinsic fluorescence spectra even though Tyr is also excited at $280 \mathrm{~nm}$. This is true also for fibrinogen despite fibrinogen containing 30 tyrosine and only 16 Trp residues. ${ }^{17}$ The fluorescence spectrum of fibrinogen in solution is shown for comparison in Supporting Information 3. Therefore, we focused on detecting Trp fluorescence emission around $350 \mathrm{~nm}$. In addition to the Trp peak, a broad peak in the range of $400-550 \mathrm{~nm}$ is almost always observed (Figure 3). Although the origin of the broad peak is not fully clear, we speculate that it is related, at least partly, to water molecules in accordance with the report by Belovolova et al. ${ }^{18}$ Indeed, the fluorescence spectra obtained from dried water exhibit the same

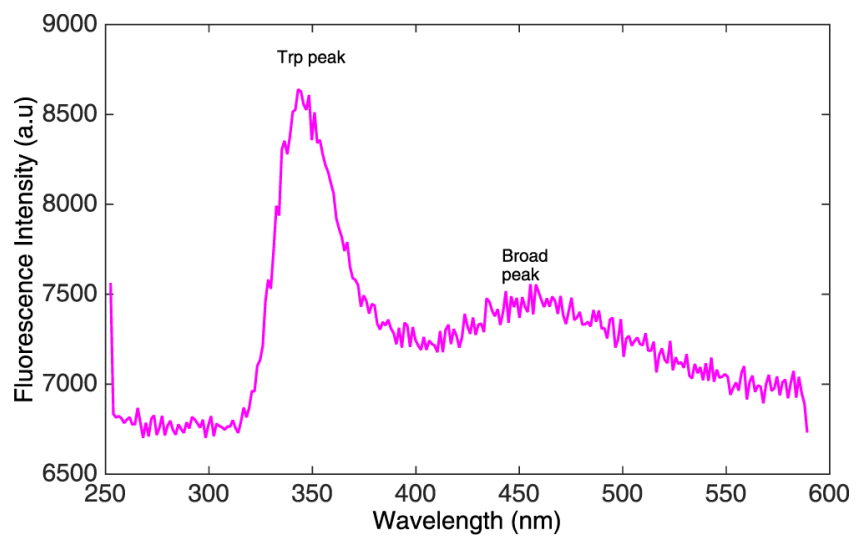

Figure 3. Fluorescence spectrum from the adsorbed fibrinogen molecules on the flat Si substrate, recorded by our setup at $280 \mathrm{~nm}$ excitation wavelength.

broad peak, see Supporting Information 4. It should be noted that the fluorescence signal in this article refers to the Trp peak.

Figure 4 shows the obtained fluorescence spectra of the fibrinogen molecules adsorbed to the arrays of nanoholes with

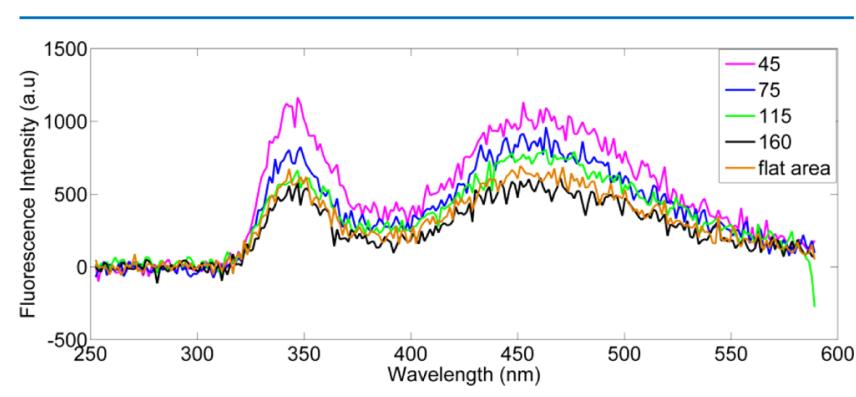

Figure 4. Fluorescence spectra of a monolayer of fibrinogen molecules adsorbed to the four nanostructured regions, the arrays of nanoholes with diameters of $45,75,115$, and $160 \mathrm{~nm}$. The brown curve indicates the obtained fibrinogen fluorescence spectrum from the flat area around the nanostructured regions.

four different diameters. There is no detectable wavelength shift of the Trp emission peak between the different spectra with the present signal to noise ratio, which makes it impossible to extract information about the conformational changes in fibrinogen molecules due to interaction with the nanoholes.

Although there was no shift in the Trp peak, the influence of nanoholes of different sizes on the fibrinogen binding can still be investigated by comparing the intensities of the Trp peak in different regions of the nanoholes. In other words, the fluorescence intensity in our measurements (Trp peak intensity) is assumed to be proportional to the amount of fibrinogen adsorption, that is, higher Trp peak intensity represents more fibrinogen adsorption. Therefore, we can observe relative changes in fibrinogen adsorption by comparing fluorescence intensities from different arrays of nanoholes, measured under identical conditions.

The fluorescence measurements in Figure 4 demonstrate that fibrinogen adsorption is influenced by the surface nanotopography. Fibrinogen molecules tend to bind more to the regions containing the arrays of 45 and $75 \mathrm{~nm}$ holes compared with the flat area around. An incremental trend in fibrinogen adsorption is observed as the diameter of the nanoholes is decreased, with the adsorption being significantly increased in 
the array of $45 \mathrm{~nm}$ holes compared with the array of $75 \mathrm{~nm}$ holes.

To explain the origin of the different binding tendencies of the fibrinogen molecules to nanoholes with different diameters, we discuss three possible hypotheses. First, the etched areas, which are the nanoholes, might have different surface properties compared with the unprocessed areas. However, the fluorescence signal enhancement, and thus the fibrinogen adsorption, does not scale with the amount of etched area.

The amount of sidewalls in the arrays is increased as the diameter of the nanoholes is decreased. Our second hypothesis, therefore, is that there might be a correlation between fibrinogen adsorption and the amount of sidewalls available for proteins to bind to. According to the AFM cross-sectional measurements, fibrinogen molecules clearly bind to the sidewalls of the arrays of 45 and $75 \mathrm{~nm}$ holes, in addition to the bottom and the flat space between nanoholes. Thus, we speculate that the additional fluorescence observed in the arrays of 45 and $75 \mathrm{~nm}$ holes might be correlated to the proteins attached to the sidewalls. To quantify the additional fluorescence, due to proteins bound to the sidewalls, we have therefore subtracted the fluorescence spectrum of the flat area from the spectrum of each specific region. The result is shown in Figure 5.

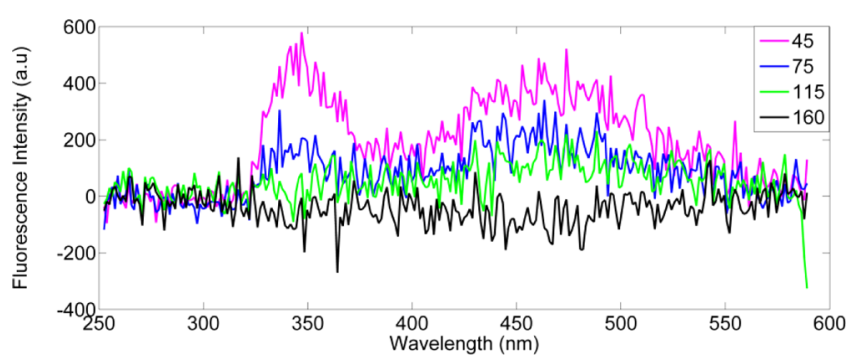

Figure 5. Fluorescence spectra of a monolayer of fibrinogen molecules adsorbed to the arrays of $45,75,115$, and $160 \mathrm{~nm}$ holes with subtracted background fluorescence (background fluorescence refers to the fluorescence spectrum of the flat area in between two adjacent nanostructured regions).

Although the fluorescence signal is enhanced, as more sidewalls are available for the proteins to bind (as the diameter of the nanoholes is decreased), the increase in fluorescence signal is much larger than the increase in the amount of sidewalls. For instance, there is a $30 \%$ increase in the amount of sidewalls available in the array of $45 \mathrm{~nm}$ holes compared with the array of $75 \mathrm{~nm}$ holes, however, the fluorescence intensity is increased by at least a factor of 2 . In other words, the Trp peak intensity is changed faster than the amount of available sidewalls. Therefore, it seems that other factors affect fibrinogen adsorption in smaller nanoholes, in addition to more available sidewalls in these regions.

We suggest the main reason for the enhanced fluorescence signal in smaller nanoholes is that fibrinogen adsorption is influenced by the curvature of the nanoholes when the diameter of the nanoholes is reduced to 45 and $75 \mathrm{~nm}$. A tentative explanation is that as the diameter decreases, and thereby the negative curvature increases, the possible contact area in between the protein molecule and the sidewalls of the nanoholes increases, leading to stronger protein binding. In the case of fibrinogen, it is unlikely that the rod is bent horizontally on the sidewalls, but rather is adsorbed standing upto the sidewalls. The diameter of the protein is then $6-9 \mathrm{~nm}$, which is similar to the diameter of, for example, serum albumin. An opposite size dependence has been seen in the interaction between nanoparticles and proteins, in which case, an increased diameter of the nanoparticles leads to an increased contact area. $^{8}$

\section{CONCLUSIONS}

We have developed a method to study the influence of nanostructured surfaces on protein binding. Constructing welldefined nanostructured surfaces, development of a monolayer of proteins on the surface (confirmed by XPS), and employment of fluorescence spectroscopy, using the intrinsic fluorescence of proteins, combined with AFM is a promising approach to investigate the effect of nanoscale surface topography on protein binding. Using the intrinsic fluorescence of proteins provides direct observation of the effect of surface nanotopography on the proteins. Our results demonstrate that fibrinogen adsorption is significantly increased as the diameter of the nanoholes is decreased to $75 \mathrm{~nm}$ and specifically to 45 $\mathrm{nm}$.

\section{EXPERIMENTAL SECTION}

Substrate Fabrication. Si wafers, as a starting material, were used to create the arrays of nanoholes. Prior to any processing, $\mathrm{Si}\langle 100\rangle$ wafers were cut into pieces of $1 \times 1 \mathrm{~cm}^{2}$ in size. The $\mathrm{Si}$ substrates were cleaned by immersing in acetone for $4 \mathrm{~min}$ in an ultrasonic bath, soaking in isopropanol (IPA) for $1 \mathrm{~min}$, and drying by nitrogen gas. ZEP 520 A7 electron beam resist was spin-coated onto the Si substrate at $9000 \mathrm{rpm}$ for $60 \mathrm{~s}$. The samples were then baked on a hot plate at $160^{\circ} \mathrm{C}$ for $10 \mathrm{~min}$. The resist coating resulted in a thickness of $180 \mathrm{~nm}$, as measured by ellipsometry.

The arrays of nanoholes with different diameters were written in the e-beam resist coated Si substrates using an EBL Raith 150 with a $10 \mathrm{kV}$ electron beam. The exposed areas of the e-beam resist were developed in $o$-xylene for $2 \mathrm{~min}$ and rinsed in IPA for $30 \mathrm{~s}$ at room temperature. The patterns of wellordered arrays of nanoholes were then transferred to the underlying Si substrate by an RIE process (Oxford Instrument) in which the resist acted as a mask. We have used the RIE method due to its ability to create an anisotropic etch profile, keeping the diameter from the aperture to the bottom of the nanoholes constant. Because protein adhesion to the nanoholes is being investigated, clean nanostructured $\mathrm{Si}$ substrates are critical. Therefore, chlorine was used as the etching gas because it does not leave any traces of organic compounds on the sample. However, there is a risk of depositing organic compounds when, for instance, fluorine-based gases are used in etching process. The flow rate of the chlorine was $20 \mathrm{sccm}$ and the pressure was $5 \mathrm{mT}$ Torr during the RIE process. The arrays of nanoholes with different diameters were etched to a depth of $50 \mathrm{~nm}$ with the RIE process described above.

After the RIE, the e-beam resist was removed by soaking the Si substrates in remover 1165 for $30 \mathrm{~min}$ on the hot plate at 90 ${ }^{\circ} \mathrm{C}$. The Si substrates were then rinsed in running highly purified water (using Milli-Q system) for $5 \mathrm{~min}$. To remove all of the resist residues from the Si surface, the oxygen plasma ashing was performed 6 times, each time for $1 \mathrm{~min}$.

AFM. The depth of the nanoholes, which is referred to as the height difference between the bottom of the nanoholes and the flat surface in between the nanoholes, was measured by AFM. 
The measurements were performed by using a Digital Instruments Dimension $3100 \mathrm{AFM}$ in tapping mode using super sharp silicon tips (SSS-NCHR AFM probe from Nanosensors) and standard AFM tips (PPP-RT-NCHR AFM probe from Nanosensors). AFM was also used to ensure the nanostructured substrates are clean and that there was no resist residue on the Si surface.

Fluorescence Setup. Fluorescence measurements were carried out using a spectrometer with $0.27 \mathrm{~m}$ focal length, connected with an UV enhanced charge-coupled device camera. The camera was cooled to $-110{ }^{\circ} \mathrm{C}$ during the measurements. A $150 \mathrm{~W}$ Xenon lamp was used as the excitation source. A 280 $\mathrm{nm}$ band pass and a short pass filter were used to remove the undesired wavelengths. The emission light from the sample was collected by a NUV 50 $\times$ objective (numerical aperture, NA = 0.42 ) and guided through lenses and mirrors to reach the spectrometer. The spectrometer slit was set to $0.5 \mathrm{~mm}$. The emission spectrum was detected in the range of 250-600 nm for 300 grooves per millimeter $(\mathrm{g} / \mathrm{mm})$ grating and with 280 $\mathrm{nm}$ excitation light. The exposure time was set to $20 \mathrm{~s}$.

Preparation of Protein Solution. Human fibrinogen (Sigma-Aldrich), purified from serum, was used for this study. We have dissolved the lyophilized proteins in highly purified water (Milli-Q) to a concentration of $1 \mathrm{mg} / \mathrm{mL}$. The protein solutions were then stored in aliquots at $-20{ }^{\circ} \mathrm{C}$ until use.

Sample Preparation. The nanostructured Si substrate was incubated with fibrinogen dissolved in water (Milli-Q) for 5 min, and immersed immediately in water (Milli-Q), for $1 \mathrm{~min}$ to remove the excess proteins. The sample was dried with nitrogen gas.

Fluorescence Measurement of Fibrinogen in Solution. The fluorescence emission spectrum between 250 and $600 \mathrm{~nm}$ of fibrinogen, approximately $50 \mu \mathrm{g} / \mathrm{mL}$ in $\mathrm{H}_{2} \mathrm{O}$, was obtained on a LS 50B spectrometer, Perkin Elmer. The excitation wavelength was $280 \mathrm{~nm}$, slits $2.5 \mathrm{~nm}$, and the scanning speed $50 \mathrm{~nm} / \mathrm{min}$. The reported data is the average of 10 scans, see Supporting Information 3.

Quantification of the Adsorbed Protein Molecules by XPS. XPS, a surface-sensitive analysis technique, was used to measure the thickness of the adsorbed fibrinogen layer on the flat Si substrate. Si $2 p$ core-level spectra were acquired at synchrotron beamline I311 of the MAX IV Laboratory in Lund, Sweden. By comparing the intensity of the Si signal for photon energies varying between 200 and $700 \mathrm{eV}$, the attenuation due to the thickness of the fibrinogen layer could be determined. For this, we assumed that the value for the inelastic mean free path of human fibrinogen should be similar to that of methionine or a $\mathrm{C}_{107} \mathrm{H}_{197} \mathrm{~N}_{29} \mathrm{O}_{49} \mathrm{~S}_{2}$ protein ${ }^{19}$ (see Supporting Information 1). The measured value of the thickness of the adsorbed fibrinogen to the flat Si surface was $4-5 \mathrm{~nm}$.

\section{ASSOCIATED CONTENT}

\section{S Supporting Information}

The Supporting Information is available free of charge on the ACS Publications website at DOI: 10.1021/acsomega.7b00241.

$\mathrm{X}$-ray photoemission spectroscopy results and data analysis; additional AFM cross-sectional profiles; fluorescence spectrum of fibrinogen in $\mathrm{H}_{2} \mathrm{O}$; fluorescence spectrum of air-dried water; fluorescence spectrum of bare Si (PDF)

\section{AUTHOR INFORMATION}

\section{Corresponding Author}

*E-mail: dan.hessman@ftf.lth.se.

ORCID

Rainer Timm: 0000-0001-8914-5924

Dan Hessman: 0000-0001-8000-368X

\section{Present Address}

${ }^{\perp}$ Applied Surface Chemistry, Chalmers University of Technology, 41296 Göteborg, Sweden (B.M.).

\section{Notes}

The authors declare no competing financial interest.

\section{ACKNOWLEDGMENTS}

The authors acknowledge financial support from NanoLund to use the Lund Nano Lab facility. Part of the work was supported by the Swedish Research Council (VR). The authors would like to thank K. Thanell for support at beamline I311 of the MAX IV Laboratory.

\section{REFERENCES}

(1) Galli, C.; Coen, M. C.; Hauert, R.; Katanaev, V.; Gröning, P.; Schlapbach, L. Creation of nanostructures to study the topographical dependency of protein adsorption. Colloids Surf., B 2002, 26, 255-267.

(2) Lord, M. S.; Foss, M.; Besenbacher, F. Influence of nanoscale surface topography on protein adsorption and cellular response. Nano Today 2010, 5, 66-78.

(3) Hulander, M.; Lundgren, A.; Berglin, M.; Ohrlander, M.; Lausmaa, J.; Elwing, H. Immune complement activation is attenuated by surface nanotopography. Int. J. Nanomed. 2011, 6, 2653.

(4) Koegler, P.; Clayton, A.; Thissen, H.; Santos, G. N. C.; Kingshott, $P$. The influence of nanostructured materials on biointerfacial interactions. Adv. Drug Delivery Rev. 2012, 64, 1820-1839.

(5) Dobrovolskaia, M. A.; Patri, A. K.; Zheng, J.; Clogston, J. D.; Ayub, N.; Aggarwal, P.; Neun, B. W.; Hall, J. B.; McNeil, S. E. Interaction of colloidal gold nanoparticles with human blood: effects on particle size and analysis of plasma protein binding profiles. Nanomedicine 2009, 5, 106-117.

(6) Jiang, W.; Kim, B. Y.; Rutka, J. T.; Chan, W. C. Nanoparticlemediated cellular response is size-dependent. Nat. Nanotechnol. 2008, $3,145-150$.

(7) Lundqvist, M.; Sethson, I.; Jonsson, B.-H. Protein adsorption onto silica nanoparticles: conformational changes depend on the particles' curvature and the protein stability. Langmuir 2004, 20, 10639-10647.

(8) Song, W.; Chen, H. Protein adsorption on materials surfaces with nano-topography. Chin. Sci. Bull. 2007, 52, 3169-3173.

(9) Riedel, M.; Müller, B.; Wintermantel, E. Protein adsorption and monocyte activation on germanium nanopyramids. Biomaterials 2001, 22, 2307-2316.

(10) Armstrong, J. K.; Wenby, R.; Meiselman, H.; Fisher, T. The hydrodynamic radii of macromolecules and their effect on red blood cell aggregation. Biophys. J. 2004, 87, 4259-4270.

(11) Chang, T. Proximity effect in electron-beam lithography. J. Vac. Sci. Technol. 1975, 12, 1271-1275.

(12) Hemmersam, A. G.; Foss, M.; Chevallier, J.; Besenbacher, F. Adsorption of fibrinogen on tantalum oxide, titanium oxide and gold studied by the QCM-D technique. Colloids Surf., B 2005, 43, 208-215.

(13) Baró, A. M.; Reifenberger, R. G. Atomic Force Microscopy in Liquid: Biological Applications; Wiley-VCH: Weinheim, 2012.

(14) Eftink, M. R. Intrinsic Fluorescence of Proteins. In Topics in Fluorescence Spectroscopy; Lakowicz, J. R., Ed.; Kluwer Academic: New York, 2000; Vol. 6, pp 1-15.

(15) Lakowicz, J. R. Principles of Fuorescence Spectroscopy; Springer: New York, 2007. 
(16) Reynolds, D. M. Rapid and direct determination of tryptophan in water using synchronous fluorescence spectroscopy. Water Res. 2003, 37, 3055-3060.

(17) Teale, F. The ultraviolet fluorescence of proteins in neutral solution. Biochem. J. 1960, 76, 381.

(18) Belovolova, L.; Glushkov, M.; Vinogradov, E.; Babintsev, V.; Golovanov, V. Ultraviolet fluorescence of water and highly diluted aqueous media. Phys. Wave Phenom. 2009, 17, 21-31.

(19) Tan, Z.; Xia, Y.; Zhao, M.; Liu, X. Electron stopping power and inelastic mean free path in amino acids and protein over the energy range of 20-20000 eV. Radiat. Environ. Biophys. 2006, 45, 135-143. 